\title{
Features of digital signal processing in the information control systems of multipositional radar
}

\author{
I.R. Parhomey a, J.M. Boiko b,*, O.I. Eromenko c \\ a Technical Cybernetics Department, National Technical University of Ukraine, Igor Sikorsky \\ Kyiv Polytechnic Institute, Peremogy 37, 03056, Kyiv, Ukraine \\ b Department of Telecommunications and Radio Engineering, Khmelnytskyi National University, \\ Institutskaya 11, 29016, Khmelnytskyi, Ukraine \\ c Department of Physics and Electrical Engineering, Khmelnytskyi National University, \\ Institutskaya 11, 29016 Khmelnytskyi, Ukraine \\ *Corresponding e-mail address: boiko_julius@ukr.net
}

\section{ABSTRAGT}

Purpose: This paper presents an algorithm for measuring angular coordinates of objects in the air radar multipositional information control systems in the application of digital microwave a digital HF amplifier in a multipositional system, which is a digital flat antenna array equidistantly.

Design/methodology/approach: The algorithm is designed to be factors determining the coordinates of air objects. Conducted a visualization of multi-synthesis process information and control system radar. Synthesized chart the direction of multi-mode radar operation for transmission and reception.

Keywords: Radar; Antenna array; Aperture; Amplifier in a multi positional system

\section{Reference to this paper should be given in the following way:}

I.R. Parhomey, J.M. Boiko, O.I. Eromenko, Features of digital signal processing in the information control systems of multipositional radar, Journal of Achievements in Materials and Manufacturing Engineering 77/2 (2016) 75-84.

\section{ANALYSIS AND MODELLING}

\section{Introduction}

A considerable attention is now being paid to multipositional radar stations (MRS) with equidistant flat EFDAA is a multichannel analyzer of radar situation in the MRS activity area [1,2]. The multipositional system is builtusing several MRS with EFDAA and special algorithms to measure parameters of signals reflected from an air target
(AT). EFDAA is a high frequent (HF) information and control device, which determines the amplitude and phase of the echo signal from any direction. So EFDAA can be considered as a digital HF amplifier (DHFA) in a multipositional system (MPS) of the determination of AT coordinates.

The algorithm of AT angular coordinate measurement of the in MPS with EFDAA-DHFA is developed under the condition that EFDAA: 
- forms the high beam (HB) in the direction of the angular coordinates AT $\theta, \varphi)$ in the mode of operation MRS of the radar transmission;

- determines the ratio of signal-to-noise $S / N$ using DHFA in the mode of radar operation on the reception;

- determines the amplitude of the signal $U_{i j}(\theta, \varphi)$ and its phase $\Phi_{i j}(\theta, \varphi)$ using DHFA in the process of digital processing of signals;

- provides a measurement of the angular coordinates of the $\operatorname{AT}(\theta, \varphi)$.

Viewing EFDAA in the form of DHFA can be possible when the measured amplitude and phase of the signal reflected from AT, the angular coordinates need to be measured, can be determined. To do this EFDAA with DHFA should be classified with certain assumptions.

Firstly, while determining angular coordinates of the AT in Cartesian $(\mathrm{X}, \mathrm{Y}, \mathrm{Z})$ or spherical $(R, \Theta, \varphi)$ coordinate systems using EFDAA, which has the amplitude and phase of the HB, it is possible to carry out the measurement of angular coordinates of the AT with the help of amplitude-phase HB EFDAA. Secondly, while defining the amplitude-phase field distribution over the aperture EFDAA using amplitude-phase $\mathrm{HB}$ it becomes possible to control the amplitude and phase of the signal in the channel of each radiator of the antenna array in the given coordinate systems $\mathrm{X}, \mathrm{Y}, \mathrm{Z}$ and $\mathrm{R}, \theta, \varphi$ and for DHFA. It is necessary to define the parameters of amplitude and phase of the $\mathrm{HB}$, the parameters of the amplitude-phase field distribution over the aperture EFDAA and parameters of the amplitude and phase of the signals in the channels of each radiator of the antenna system (in the given coordinate systems $\mathrm{X}, \mathrm{Y}, \mathrm{Z}$ and $(R, \theta, \varphi)$ and for DHFA. Thirdly, in the case of identifying the parameters of HB amplitude and phase, the parameters of amplitude-phase field distribution over the aperture EFDAA and parameters of the amplitude and phase of the signals in the channels of each radiator EFDAA (coordinates $\mathrm{X}, \mathrm{Y}, \mathrm{Z}$ or $R, \theta, \varphi$ ) the distribution of voltage signals $U_{i j}(\theta, \varphi)$ and their phases $\Phi_{i j}(\theta, \varphi)$ through the receiving channels EFDAA-DHFA will determine the AT angular coordinates. For this purpose it is necessary only to measure these parameters in relation to the phase centers of radiators as separate channels for addition of signals with voltage $U_{i j}(\theta, \varphi)$ and phase $\Phi_{i j}(\theta, \varphi)$.

The purpose of this research is the formalization of the algorithm of angular coordinate measurement of air objects in radar multipositional information and control systems when applying digital highly frequent amplifier and automatic monitoring of the angular distribution of the field radiation phase component of EFDAA-DHFA.

To achieve this goal it is necessary to solve the following tasks:
- to develop a systematic methodology of algorithm of angular coordinates of measurement of air objects in multipositional radar information and control systems;

- to conduct synthesis of multipositional information and control systems of radar stations with equidistant flat digital antenna arrays using the proposed algorithm.

\section{Developing an algorithm measuring angular coordinates air targets}

The algorithm of switching and assembling the signals with $U_{i j}(\theta, \varphi)$ voltage and $\Phi_{i j}(\theta, \varphi)$ phase in accordance with the target functions $\max U_{\mathrm{ij}}(\theta, \varphi)$ and $\min \Phi_{i j}(\theta, \varphi)$, which control the measurement of the AT angular coordinates, is the third part of the algorithm of AT angular coordinate measurement while using EFDAA-DHFA.

Those three presented parts of the algorithm of AT angular coordinate measurement using EFDAA can be implemented as one algorithm of measurement of AT angular coordinates using DHFA, which enters into the circuit of MRS.

Structural diagram of the algorithm of measurement of AT angular coordinates when using EFDAA is shown in Fig. 1, and the structural diagram of DHFA - in Figure 2. Structural diagram of the algorithm of AT angular coordinate measurement when using EFDAA-DHFA contains three parts of successive calculation of AT angular coordinates. According to this, it is necessary to develop the structural diagram of DHFA, which will exactly suit the sequence of calculation of measurement of angular coordinates in accordance with the above algorithm and the structural diagram of the antenna system of receiving and processing the signals at $\mathrm{HF}$ and in the circuit of MRS.

The HF part of EFDAA-DHFA, which is shown in Fig. 2, contains radiators, multi-channel HF device of signal distribution, the channels of receiving the signals with the selection of signals with the $U_{i j}(\theta, \varphi)$ voltage and $\Phi_{i j}(\theta, \varphi)$ phase. Low-frequency (LF) part of EFDAADHFA contains the devices of signal processing and interfacing with a microprocessor of signals which carries out the measurement of AT angular coordinates in the circuit of MRS. These two parts are the basis of DHFA.

To identify the structural diagram of EFDAA and DHFA it is necessary to undertake the analytical formalization of the processes of AT angular coordinate measurement in these devices and their comparison. The first part of the structural diagram of the algorithm is formalized in the following way. The plane of the aperture of EFDAA is located in the plane of XOY (the size is 
limited by the coordinates $\mathrm{X} 1 \mathrm{X} 2$ - Y1Y2), and in the spherical system with coordinates $R, \theta, \varphi$ has an axle $\mathrm{OZ}$ (Fig. 3) at point $\mathrm{O}$ (the origin of coordinates).

HB EFDAA is formed with a flat revelation of X1X2 Y1Y2 in the direction of the AT in accordance with the task decision of the mixed synthesis of antennas.

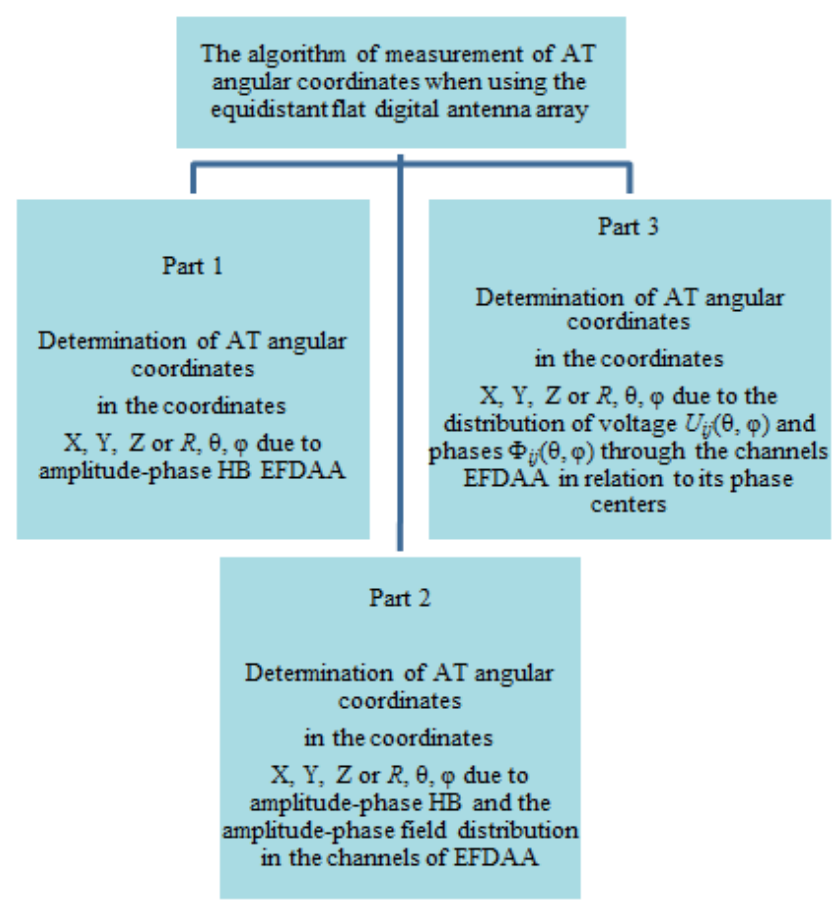

Fig. 1. The structural scheme of the algorithm of measurement of angular coordinates of the air targets when using the equidistant flat digital antenna array

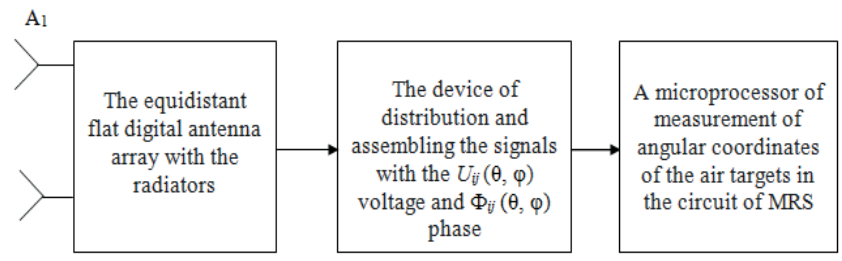

Fig. 2. The structural scheme of the digital HF of the amplifier in the circuit

According to [3], there are four possible goals of the mixed synthesis of antenna, when the following data is set: $\mathrm{HB}$ amplitude and the amplitude of the current in EFDAA; $\mathrm{HB}$ amplitude and phase of the current in EFDAA; HB phase and the amplitude of the current in EFDAA; HB phase and phase of the current in EFDAA.

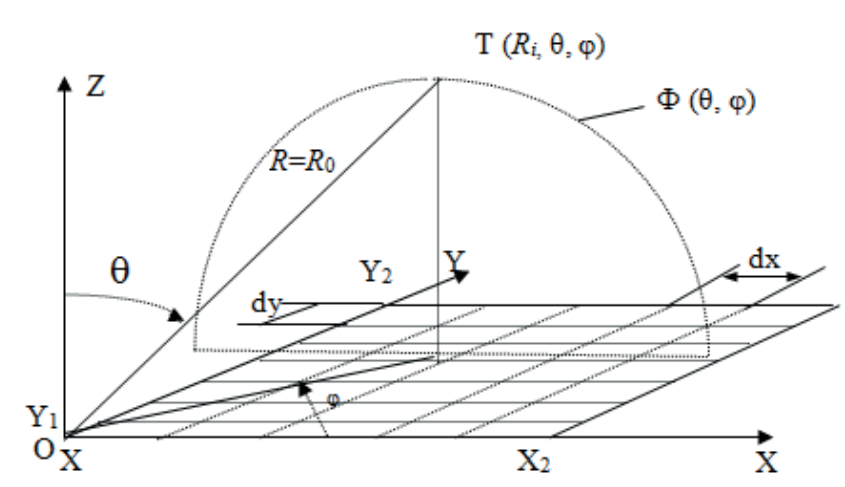

Fig. 3. Determination of target coordinates while using the equidistant flat digital antenna array

There are root-mean-square deflections of a given HB $|f(U)|$ from the $\mathrm{HB}$, which was obtained $\left|f(U)^{\prime}\right|$ in the process of solving, and which is supposed to arrive at the appropriate HB EFDAA. The measurement of AT angular coordinates can be carried out by using the amplitudephase HB EFDAA under the condition of identification of its parameters and settings DHFA. Therefore, two problems should be solved.

Task 1. The amplitude of the HB $|f(U)|$ and the current amplitude $A(\xi)$ are given:

$J\left(\psi_{i}(\xi)\right)=\int_{u_{1}}^{u_{2}}\left[f_{\text {set }}^{2}(U)-\left(\int_{-\sigma}^{\sigma} A(\xi) \cos \left(U \xi+\psi_{i}(\xi) d \xi^{2}\right)-\right.\right.$

$-\left(\int_{-\sigma}^{\sigma} A(\xi) \sin \left(U \xi+\psi_{i}(\xi) d \xi^{2}\right)\right]^{2} d U$

Task 2. The HB $|\Phi(U)|$ phase and current $\psi(\xi)$ phase are given:

$$
\begin{aligned}
& J\left(A_{i}(\xi)\right)=\int_{u_{1}}^{u_{2}}\left[\operatorname{tg} \Phi_{\text {set }}^{2}(U)-\left(\int_{-\sigma}^{\sigma} A_{i}(\xi) \cos \left(U \xi+\psi(\xi) d \xi^{2}\right)-\right.\right. \\
& -\left(\int_{-\sigma}^{\sigma} A_{i}(\xi) \sin \left(U \xi+\psi(\xi) d \xi^{2}\right)\right]^{2} d U .
\end{aligned}
$$

The process of approximation in the given tasks should be done in such a way that each next function of the set values of amplitude and phase will reduce the change of the functional $\delta J(.$.$) according to [3-11]. Under the terms of$ the AT angular coordinate measurement in EFDAA it is required to use the change of the functional $\delta J(\ldots)$ in the problem 2, when HB phase $|\Phi(U)|$ and current $\psi(\xi)$ phase are set. Then, according to [3]: 
$\delta J(\ldots)=-\varepsilon \int_{-\sigma}^{\sigma} \eta_{i}(\xi) F(\xi) d \xi$

$$
A_{i+1}(\xi)=A_{i}(\xi)+\varepsilon \eta(\xi)
$$

In this case the choice of the starting approximation has an essential meaning. This requires the use of a linear equation system in the problems with the given $\mathrm{HB} \Phi(U)$ phase and the distribution of current $\psi(\xi)$ phase. Then, according to [3-11], in the equation:

$$
\operatorname{tg} \Phi_{\text {set }}(U)=\frac{\int_{-\sigma}^{\sigma} A(\xi) \sin (U \xi+\psi(\xi) d \xi)}{\int_{-\sigma}^{\sigma} A(\xi) \cos (U \xi+\psi(\xi) d \xi)},
$$

the integrals must be replaced by sums. The result is:

$$
\begin{aligned}
& \operatorname{tg} \Phi_{s e t}(U)=\sum_{n=0}^{N-1 M-1} \sum_{m=0} A_{i}\left(\xi_{n}\right) \cos \left(U \xi_{n}+\psi\left(\xi_{n}\right)\right) \Delta \xi_{n} A_{j}\left(\xi_{m}\right) \times \\
& \times \cos \left(U \xi_{m}+\psi\left(\xi_{m}\right)\right) \Delta \xi_{m}-\sum_{n=0}^{N-1 M-1} \sum_{m=0} A_{i}\left(\xi_{n}\right) \sin \left(U \xi_{n}+\psi\left(\xi_{n}\right)\right) \times \\
& \times \Delta \xi_{n} A_{j}\left(\xi_{m}\right) \sin \left(U \xi_{m}+\psi\left(\xi_{m}\right)\right) \Delta \xi_{m} .
\end{aligned}
$$

Having determined $\mathrm{HB} \Phi(U)$ phase at several points $U_{1}, U_{2}, \ldots, U_{n}$, the system of homogeneous equations for $A\left(\xi_{1}\right), A\left(\xi_{2}\right), \ldots, A\left(\xi_{n}\right)$ will be obtained, and upon that it is necessary to introduce the additional inhomogeneous equation (with the requirement of regulation) and solve the inhomogeneous system of equations.

\section{The development of a systematic methodology of algorithm of measurement of angular coordinates of air objects}

When determining $\mathrm{HB} \Phi(\mathrm{U})$ phase EFDAA in the mode of MRS on admission the HB amplitude $|\mathrm{f}(\mathrm{U})|$ and the amplitude of the current $A(\xi)$ EFDAA in the mode of MRS in a transmission according to tasks 1 and mode DHFA should be determined. It is required to determine the maximum HB EFDAA in the direction of the target with the angular coordinates of AT $\theta, \varphi$ in the MRS mode, of the transmission and measure the amplitude of the signal in the mode of DHFA. Then, based on the principle of reciprocity of antenna theory it is possible to determine the amplitude of the HB $|f(U)|$ and the amplitude of the current $A(\xi)$ EFDAA in the mode of MRS on the reception, and from it to proceed to phase $\mathrm{HB} \Phi(U)$ of the antenna array.
According to [3] the amplitude characteristic of $\mathrm{HB}$ EFDAA with the measurement of the angular components of the field along the AT angular coordinates has two components:

$$
\begin{aligned}
& \mathrm{E}^{\theta}=i \frac{\exp (-j k R)}{2 \lambda R}\left[\frac{z_{0}}{\alpha} \cos \theta \sin \varphi N_{X}-\left(\cos \varphi+\frac{z_{0}}{\alpha} \sin \theta\right) N_{Y}\right] \\
& \left.\mathrm{E}^{\varphi}=i \frac{\exp (-j k R)}{2 \lambda R}\left[\left(\sin \theta+\frac{z_{0}}{\alpha} \cos \varphi\right) N_{X}-N_{Y} \cos \theta \sin \varphi\right)\right] .
\end{aligned}
$$

In the simplified version, the $\mathrm{HB} \Phi(U)$ phase of EFDAA can be considered as the angular distribution of the phase component of the radiation field. $\Phi(\theta, \varphi)$ can be considered instead of the function $\Phi(U)$, which has the equiphase surfaces of the EFDAA field emission in space:

$$
F(\theta, \varphi)=R_{0}+\Phi(\theta, \varphi) / k
$$

where $R_{0}$ is the radius of a sphere with the centre of coordinate system, which is used in calculations of the EFDAA field in the far zone; $k=2 \pi / \lambda$ - is the wave number; $\lambda$ - the length of the wave.

When EFDAA will be located accordingly with Fig. 3.

$\Phi(\theta, \varphi)=k\left(x_{0} \sin \theta \cos \varphi+y_{0} \sin \theta \sin \varphi+z_{0} \cos \theta+a\right)$,

where $x_{0}, y_{0}, z_{0}$ - is the start of coordinates relatively to EFDAA; $a$ - is a constant.

The surface $\Phi(\theta, \varphi)$ is a sphere, and a phase EFDAA centre coincides with the centre of the sphere. There won't be any coincidence while a random selection of the starting of coordinates, therefore, the conclusion about the presence of the phase centre is being made by the fact or it can be found by moving the origin of EFDAA coordinates [6-10].

Regardless this, the phase characteristic $\Phi(\theta, \varphi)$ of $\mathrm{HB}$ EFDAA could be introduced if to register its wave field with the intensity renewal according to [3,6-11],

$F(\theta, \varphi)=|F(\theta, \varphi)| \exp j(\arg F(\theta, \varphi))$,

the reconstruction of the wave field of EFDAA is being carried out by using the Hilbert transformation from the $\ln |F(\theta, \varphi)|$ function:

$\arg F(\theta, \varphi)=\frac{1}{\pi} \oint \int_{-\infty}^{\infty} \frac{\ln \left|F(\theta, \varphi)^{\prime}\right|}{(\theta, \varphi)^{\prime}-(\theta, \varphi)} d \theta^{\prime} d \varphi^{\prime}+\sum \arg \frac{z-z^{\prime}}{z-z_{i}}$, 
where $z_{i}$ - is the complex zeros of the function $|F(z)|$, which is an analytic continuation of $F(\theta, \varphi)$ in the complex variable plane; $|f(U)|$ - is a spatial spectrum of the wave field, which is formed of the conclusive distribution of a field in coordinates $\mathrm{X}, \mathrm{Y}$ simultaneously [3]. In this case $\theta=k x / z$ is the angular spatial frequency of the field on the $\mathrm{X}$-axis, $\varphi=k y / z$ is the angular spatial frequency on the Y-axis.

The relationship between the amplitude and phase of the wave field as in EFDAA as DHFA is set, based on the ratio [3].

$\ln F(\theta, \varphi)=\ln |F(\theta, \varphi)|+j \operatorname{arctg} F(\theta, \varphi)$,

Check of connection of real and imaginary parts of the function $F(\theta, \varphi)$ is made under the conditions of CauchyRiemann [3-11]:

$$
\begin{aligned}
& \frac{\partial}{\partial \theta} \ln |F(\theta, \varphi)|=\frac{\partial}{\partial \varphi} \arg F(\theta, \varphi) \\
& \frac{\partial}{\partial \varphi} \ln |F(\theta, \varphi)|=-\frac{\partial}{\partial \theta} \arg F(\theta, \varphi) .
\end{aligned}
$$

The connection between the amplitude and phase of $\mathrm{HB}$ EFDAA is being established as a result of a finding $\arg F(\theta, \varphi)$ by [3] that correspond with the connection between the amplitude and phase of the signal in DHFA.

According to the analysis of EFDAA-DHFA work it is possible to draw a conclusion about the identification of their structural diagrams according to the algorithm of AT angular coordinate measurement in the circuit of MRS at HF.

The system development methodology of algorithm of AT angular coordinate measurement using EFDAA-DHFA is made on the basis of partial diagrams.

In this case, the approximation of a given HB EFDAADHFA is carried out using the special functions (the partial diagrams), and exact solution of the synthesis problem for each of them can be found, i.e., the synthesized amplitude in HB EFDAA-DHFA in the mode of MRS at transmission (task 1, Figs. 4, 5). Search of distribution of AT radiation sources is in the form of a superposition of partial field distributions of EFDAA-DHFA, which are controlled by the separate partial diagram radiators, so HB EFDAA-DHFA is synthesized in the mode of MRS at receiving (task 2, Fig. 5). Considering the conditions in [3], the system of partial diagrams is chosen while solving the problem 2 for finding the functional $J\left(A_{i}(\xi)\right)$.

In this case, HB EFDAA-DHFA:

$|f(U)|=f(U) f 1(U)$, where $f(U)$ - HB of one EFDAA-DHFA radiator; $|f 1(U)|$ HB of EFDAA-DHFA multiplier.

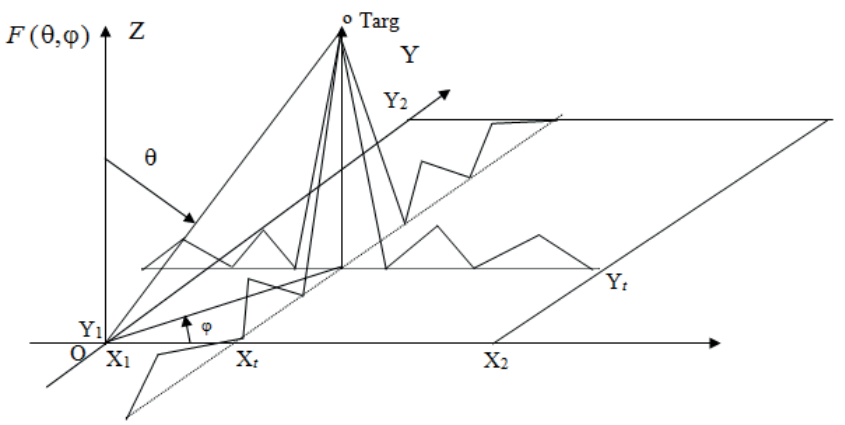

Fig. 4. The synthesis of HB EFDAA-DHFA in the mode of MRS

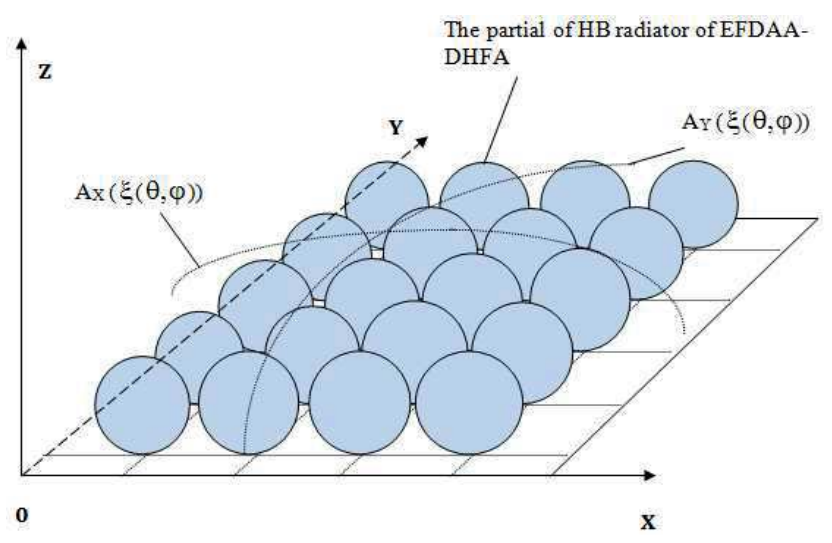

Fig. 5. The synthesis of HB EFDAA-DHFA in the mode of MRS for transmission - reception (fragment)

The amplitude HB of each EFDAA-DHFA radiator is "carded", which is "pressed" from the XOY plane and has the appearance of a perfect "circle".

HB of EFDAA-DHFA multiplier is determined by the expression:

$|f 1(U)|=\sum_{n=0}^{N-1} \sum_{m=0}^{M-1} a_{n} a_{m} e^{i j k\left(\rho_{n}+\rho_{m}\right) R_{i} R_{j}}$,

where $n, m$ - are the ordinal numbers of radiators; $a_{\mathrm{n}}, a_{m}$ are the coefficients of proportionality of complex amplitude of $\mathrm{n}$ and m's radiator current; $\mathrm{N} \times \mathrm{M}$ are the number of radiators concerning two EFDAA-DHFA coordinates; $\rho_{n}, \rho_{m}$ - radius-vector that defines the position of the centre of the $\mathrm{n}$ and m's radiators of EFDAA-DHFA; $R_{i}=R / R, R_{j}=R / R^{\prime /}$ - the radius-vectors of observation points by two coordinates (see Fig. 3). 


\section{Visualization of the synthesis process of the multipositional information management system of radarstation}

The degree of approximation of two functions $|f(U)|$ and $F(\theta, \varphi)$ in two planes for two targets is determined by magnitude [1].

$\delta_{t}=\int_{-\pi}^{\pi} P\left(U_{n}\right)\left[F\left(U_{n}\right)-\left.\left|f\left(U_{n}\right)\right|\right|^{2} d U_{n}\right.$

where $P\left(U_{n}\right)$ is a weight function that governs the degree of approximation $|f(U)| \approx F(\theta, \quad \varphi) \cong F\left(U_{n}\right)$ for the different angular directions of observation, for example 2 targets (Fig. 6), on the magnitudes $\delta_{t}^{1}$ i $\delta_{t}^{2}$.

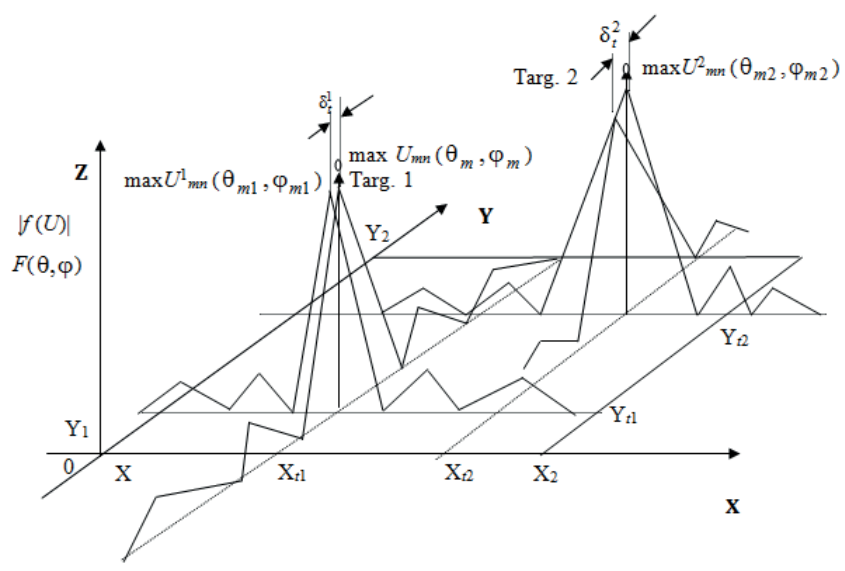

Fig. 6. HB EFDAA-DHFA in the mode of MRS at reception

The AT coordinate measuring using EFDAA-DHFA should be carried out in the mode of "transmission reception". Therefore, it is necessary to analyze the adequacy of the measurement process the real AT angular coordinate measurement in MRS from EFDAA-DHFA, when determining the maximum signal-to-noise ratio $S / N$ with the given spatial direction of $\mathrm{HB} \theta_{m n}, \varphi_{n m}$ of finding the goal and concurrently the angular coordinates that correspond to this maximum $S / N$.

The estimated power ratios between signals and noise is carried out using max $S / N$ for given probabilities of detection and false alarm, and measurement precision of target angular coordinates and its gear in MRS with EFDAA-DHFA, when the max HB is set in the direction of the target [12-18]:

$$
\begin{aligned}
& \max S / N\left(\theta_{m n}, \varphi_{m n}\right)=\frac{P_{T}\left(\theta_{m n}, \varphi_{m n}\right) G_{T}\left(\theta_{m n}, \varphi_{m n}\right) \times}{(4 \pi)^{3} k T R_{1}^{2}\left(\theta_{m n}, \varphi_{m n}\right) F_{1}\left(\theta_{m n}, \varphi_{m n}\right) \times} \\
& \frac{\times G_{R}\left(\theta_{m n}, \varphi_{m n}\right) \lambda^{2} \sigma\left(\theta_{m n}, \varphi_{m n}\right) \tau\left(\theta_{m n}, \varphi_{m n}\right)}{\times R_{2}^{2}\left(\theta_{m n}, \varphi_{m n}\right) F_{2}\left(\theta_{m n}, \varphi_{m n}\right) L\left(\theta_{m n}, \varphi_{m n}\right)}
\end{aligned}
$$

where $P_{T}\left(\theta_{m n}, \varphi_{n m}\right)$ is the transmitter potency in the direction of the target coordinates, $\mathrm{kW} ; \mathrm{k}=1,38 \cdot 10-23 \mathrm{~J} / \mathrm{K}^{\circ}$ is the Boltzmann constant; $T\left(\theta_{m n}, \varphi_{n m}\right)=\left(T_{\mathrm{A}} / L_{R}+T_{R}+T_{E}\right)$ is the effective temperature of the receiver channel for a given direction, $\mathrm{K}^{\circ} ; T_{\mathrm{A}}\left(\theta_{m n}, \varphi_{n m}\right)$ is the noise temperature of the antenna for a given direction, $\mathrm{K}^{\circ} ; L_{R}\left(\theta_{m n}, \varphi_{n m}\right)$ is the loss between antenna and receiver for a given direction, $\mathrm{dB} ; T_{R}$ $\left(\theta_{m n}, \varphi_{n m}\right)$ is the effective noise temperature of the antenna receiver for a given direction; $T_{R}=T_{T}(1-1 / L), \mathrm{K}^{\circ} ; \Phi\left(\theta_{m n}, \varphi_{n m}\right)$ - is the phase HB of EFDAA-DHFA; $T_{T}\left(\theta_{m n}, \varphi_{n m}\right)$ - is the temperature of the line antenna-receiver for a given direction, $\mathrm{K} ; T_{E}\left(\theta_{m n}, \varphi_{n m}\right)$ is the effective noise temperature of the receiver for a given direction, $T_{E}=(N F-1) T_{n}, \mathrm{~K}^{\circ}$; $T_{n}\left(\theta_{m n}, \varphi_{n m}\right)$ - is the temperature where determine the noise coefficient $N F$ for a given direction, $\mathrm{K} ; \tau\left(\theta_{m n}, \varphi_{n m}\right)$ - is the time of the target observation for a given direction, s; $F_{1}\left(\theta_{m n}, \varphi_{n m}\right), \quad F_{2}\left(\theta_{m n}, \varphi_{n m}\right)$ - are the coefficients of radiowaves spreading for a given direction, $\mathrm{dB} ; L$ - is the coefficient of losses in antenna-fidern system for a given direction, $\mathrm{dB} ; \sigma$ - is the effective surface of target reflection for a given direction, $\mathrm{m}^{2} ; \lambda$ - is the wavelength of MRS, $\mathrm{m}$; $R_{1}^{2}, R_{2}^{2}$ - are the distance from MRS to targets and from targets to MRS, km; $G_{T}\left(\theta_{m n}, \varphi_{n m}\right)$ - is a gain of EFDAADHFA in the mode of MRS on transmission, $\mathrm{dB}$; $G_{R}\left(\theta_{m n}, \varphi_{n m}\right)$ - is a gain of EFDAA-DHFA in the mode of MRS on reception, $\mathrm{dB}$.

To determine the maximum $S / N\left(\theta_{m n}, \varphi_{n m}\right)$ is necessary to take into account the general conditions of providing max $G_{\mathrm{T}}\left(\theta_{m n}, \varphi_{n m}\right)=D\left(\theta_{m n}, \varphi_{n m} \quad\right) \eta$, where $D\left(\theta_{m n}, \varphi_{n m}\right)$ is the coefficient of directed action (CDA), and the efficiency (E) of the antenna.

The amplitude of HB EFDAA-DHFA, which satisfies the condition $\max D\left(\theta_{m n}, \varphi_{n m}\right)$, can be found by the formula:

$F\left(\theta_{m n}, \varphi_{m n}\right)=F_{R}\left(\theta_{m n}, \varphi_{m n}\right)+j F_{J}\left(\theta_{m n}, \varphi_{m n}\right)$.

The phase HB EFDAA-DHFA:

$\Phi\left(\theta_{m n}, \varphi_{m n}\right)=\operatorname{arctg}-\frac{F_{J}\left(\theta_{m n}, \varphi\right)}{F_{R}\left(\theta_{m n}, \varphi_{m n}\right)}$,

is characterized by a partial phase centre and its resistance. It is considered also that the individual radiators of 
EFDAA-DHFA have the phase centers that are determined by the coordinates of their location in the plane of XOY (see Fig. 3, 5).

The following points are taken into account:

1. EFDAA-DHFA contains $2 N+1$ rows which are parallel to $\mathrm{Y}$ axis and $2 M+1$ rows, which are parallel to $\mathrm{X}$ axis.

2. The EFDAA-DHFA radiator with the number of $m n$ has coordinates $\mathrm{xm}=\mathrm{mdx}$ and $\mathrm{yn}=\mathrm{ndy}$, so $-\mathrm{M} \leq \mathrm{m} \leq \mathrm{M}$ and $\mathrm{N} \leq \mathrm{n} \leq \mathrm{N}$.

3. The multiplier of EFDAA-DHFA has the form:

$$
F_{k}\left(\theta_{m n}, \varphi_{m n}\right)=f_{x}\left(\theta_{m n}, \varphi_{m n}\right) f_{y}\left(\theta_{m n}, \varphi_{m n}\right),
$$

where:

$$
\begin{aligned}
& f_{x}\left(\theta_{m n}, \varphi_{m n}\right)=\sum_{m=-M}^{M} I_{m} e^{i m \beta d_{x} \sin \theta \cos \varphi}, \\
& f_{y}\left(\theta_{m n}, \varphi_{m n}\right)=\sum_{n=-N}^{N} I_{n} e^{i n \beta d_{y} \sin \theta \cos \varphi}, \\
& I_{m}=I_{m 0} / I_{00}, I_{n}=I_{n 0} / I_{00},
\end{aligned}
$$

for accepted restrictions on the distribution of currents in the radiators.

4. Using the law of current distribution on the radiators:

$$
I_{m n}=I_{00} e^{-i(m \psi x+n \psi y)},
$$

the multiplier of EFDAA-DHFA is determined by the expression:

$$
\begin{aligned}
& F_{k}\left(\theta_{m n}, \varphi_{m n}\right)=I_{00}^{2}\left[\sum_{m=-M}^{M} e^{i m\left(\beta d x m \sin \theta_{m} \cos \varphi_{m}-\psi_{x}\right)}\right] \times, \\
& \times\left[\sum_{m=-N}^{N} e^{i n\left(\beta d_{y n} \sin \theta_{m} \cos \varphi_{m}-\psi_{y}\right)}\right]
\end{aligned}
$$

where $\psi_{x m}, \psi_{x n}$ - are the phase offsettings in $\mathrm{X}$ and $\mathrm{Y}$ axes between neighboring radiators.

5. EFDAA-DHFA has HB maximum in the direction of the target $1-\theta \mathrm{m} 1, \varphi n 2$ and the target $2-\theta \mathrm{m} 2, \varphi n 2$ and according to [12-15] and Fig. 5, the algorithm of the of angular coordinates measurement of targets 1 and 2 using EFDAA-DHFA determines the appropriate ratios: $\varphi_{m 1}=\operatorname{arctg}\left(\frac{\psi_{y 1} d x_{1}}{\psi_{x 1} d y_{1}}\right)$

$\theta_{m 1}=\operatorname{arctg} \sqrt{\left(\frac{\psi_{x 1}^{2}}{\beta^{2} d x_{1}^{2}}\right)+\left(\frac{\psi_{y 1}^{2}}{\beta^{2} d y_{1}^{2}}\right)}$,

$\varphi_{m 2}=\operatorname{arctg}\left(\frac{\psi_{y 2} d x_{2}}{\psi_{x 2} d y_{2}}\right)$

$$
\theta_{m 2}=\operatorname{arctg} \sqrt{\left(\frac{\psi_{x 2}^{2}}{\beta^{2} d x_{2}^{2}}\right)+\left(\frac{\psi_{y 2}^{2}}{\beta^{2} d y_{2}^{2}}\right)} .
$$

6. The formation and offsetting of the main lobes of $\mathrm{HB}$ EFDAA-DHFA accordingly objectives 1 and 2 in modes of transmission - reception is made due to the algorithm $[12,14,15]$.

$$
\begin{aligned}
& \chi_{x 1}=\beta \cos \gamma_{x 1}, \chi_{y 1}=\beta \cos \gamma_{y 1}, \\
& \chi_{x 2}=\beta \cos \gamma_{x 2}, \chi_{y 2}=\beta \cos \gamma_{y 2}, \\
& F_{K 1}\left(\chi_{X 1}, \chi_{\mathrm{Y} 1}\right)=\sum_{m=-M}^{M} \sum_{n=-N}^{N} \frac{I_{m n}^{1}}{I_{001}} e^{i m_{1} d_{x 1} x_{x 1}+i \eta_{1} d_{y 1} \chi_{y 1}}, \\
& F_{K 2}\left(\chi_{X 2}, \chi_{\mathrm{Y} 2}\right)=\sum_{m=-M}^{M} \sum_{n=-N}^{N} \frac{I_{m n}^{2}}{I_{002}} e^{i m_{2} d x_{2} \chi_{x 2}+i m_{2} d_{y 2} \chi_{y 2} .}
\end{aligned}
$$

7. Behaviour of multipliers FK1, FK2 for $0 \leq \theta \mathrm{m} \leq \pi / 2$ and $0 \leq \varphi n m \leq 2 \pi$ is taken into account in the algorithm by known methods [3,11-19].

8. CDA EFDAA-DHFA, according to $[15,16]$, towards the targets 1 and 2.

$$
\begin{aligned}
& \max D 1\left(\theta_{m n}, \varphi_{m n}\right)=\pi \cos \theta_{m n}^{1} D_{\mathrm{X} 1}\left(\theta_{m n}^{1}, \varphi_{m n}^{1}\right) D_{\mathrm{Y} 1}\left(\theta_{m n}^{1}, \varphi_{m n}^{1}\right) \\
& \max D 2\left(\theta_{m n}, \varphi_{m n}\right)=\pi \cos \theta_{m n}^{2} D_{\mathrm{X} 2}\left(\theta_{m n}^{2}, \varphi_{m n}^{2}\right) D_{\mathrm{Y} 2}\left(\theta_{m n}^{2}, \varphi_{m n}^{2}\right)
\end{aligned}
$$

9. In order to find $\max (\mathrm{S} / \mathrm{N}) 1(\theta \mathrm{mn}, \quad \varphi n m)$ and $\max (\mathrm{S} / \mathrm{N}) 2(\theta \mathrm{mn}, \varphi \mathrm{nm})$ it is necessary to express maxGT $(\theta \mathrm{mn}, \varphi \mathrm{nm})$ for the transmitting mode in such a way:

$$
\begin{aligned}
& G_{T}\left(\theta_{m}, \varphi_{m}\right)=f^{2}\left(\theta_{m}, \varphi_{m}\right)\left[\sum_{m=-M}^{M} \sum_{n=-N}^{N} \frac{I_{m n}}{I_{00}} e^{i m d_{x} x_{x}+i n d_{y} \chi_{y}}\right]^{2} \times, \\
& \times \eta\left(\theta_{m}, \varphi_{m}\right)
\end{aligned}
$$


and $\max G_{R}\left(\theta_{m n}, \varphi_{n m}\right)$ for the mode on reception:

$G_{R 1}\left(\theta_{m 1}, \varphi_{m 1}\right)=f^{2}\left(\theta_{m}, \varphi_{m}\right)\left[\sum_{m=-M}^{M} \sum_{n=-N}^{N} \frac{I_{m n}^{1}}{I_{00}^{1}} e^{i m_{1} d_{x 1} \chi_{x 1}+i n_{1} d_{y 1} \chi_{y 1}}\right]^{2}$,

$\times \eta\left(\theta_{m}, \varphi_{m}\right)$

$G_{R 2}\left(\theta_{m 2}, \varphi_{m 2}\right)=f^{2}\left(\theta_{m}, \varphi_{m}\right)\left[\sum_{m=-M}^{M} \sum_{n=-N}^{N} \frac{I_{m n}^{2}}{I_{00}^{2}} e^{i m_{2} d_{x 2} \chi_{x 2}+i n_{1} d_{y 2} \chi_{y 2}}\right]^{2}$.

$\times \eta\left(\theta_{m}, \varphi_{m}\right)$

10. It is also necessary to take into account the mutual influence of the radiators in the receiving mode, which is determined according to $[11,15]$ and Fig. 7 by the formula:

$\left[Z_{m n}\right] \cdot\left|I_{m n}\right|=\left|U_{m n}\right|$,

where $\left|Z_{m n}\right|$ is the matrix of own and mutual impedances of the radiators for each target; $\left|I_{m n}\right|$ - is a vector-column-row of complex amplitudes of the currents of the radiators in $\mathrm{X}$ or Y coordinates; $\left|U_{m n}\right|$ - is a vector-row-column of complex voltages of the radiators in $\mathrm{X}$ or $\mathrm{Y}$ coordinates.

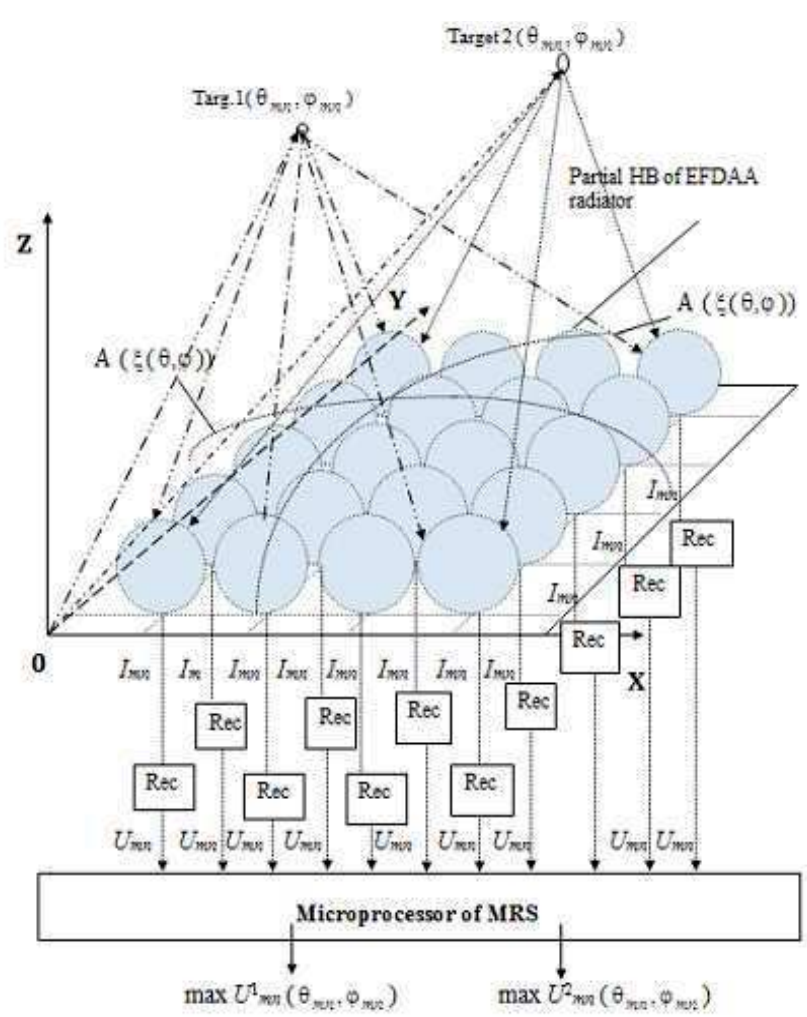

Fig. 7. EFDAA-DHFA is in the mode of MRS on reception (fragment)

For target №1: $\left|Z_{m n}^{1}\right|,\left[I_{m n}^{1}\right]$ and $\left[U_{m n}^{1}\right]$ are determined by the expressions:

$\left[Z_{m n}^{1}\right]=\left[\begin{array}{l}Z_{11}^{1}, \ldots, Z_{1 n}^{1} \\ Z_{21}^{1}, \ldots, Z_{2 n}^{1} \\ ------ \\ Z_{m 1}^{1}, \ldots, Z_{m n}^{1}\end{array}\right],\left[I_{m n}^{1}\right]=\left[\begin{array}{l}I_{11}^{1}, \ldots, I_{1 n}^{1} \\ I_{21}^{1}, \ldots, I_{2 n}^{1} \\ ------ \\ I_{m 1}^{1}, \ldots, I_{m n}^{1}\end{array}\right]$,

$\left[U_{m n}^{1}\right]=\left[\begin{array}{l}U_{11}^{1}, \ldots, U_{1 n}^{1} \\ U_{21}^{1}, \ldots, U_{2 n}^{1} \\ ------ \\ U_{m 1}^{1}, \ldots, U_{m n}^{1}\end{array}\right]$

For target №2: $\left|Z_{m n}^{2}\right|,\left[I_{m n}^{2}\right]$ and $\left[U_{m n}^{2}\right]$ accordingly:

$\left[Z_{m n}^{2}\right]=\left[\begin{array}{l}Z_{11}^{2}, \ldots, Z_{1 n}^{2} \\ Z_{21}^{2}, \ldots, Z_{2 n}^{2} \\ ------ \\ Z_{m 1}^{2}, \ldots, Z_{m n}^{2}\end{array}\right],\left[I_{m n}^{2}\right]=\left[\begin{array}{l}I_{11}^{2}, \ldots, I_{1 n}^{2} \\ I_{21}^{2}, \ldots, I_{2 n}^{2} \\ ------ \\ I_{m 1}^{2}, \ldots, I_{m n}^{2}\end{array}\right]$,

$\left[U_{m n}^{2}\right]=\left[\begin{array}{l}U_{11}^{2}, \ldots, U_{1 n}^{2} \\ U_{21}^{2}, \ldots, U_{2 n}^{2} \\ ------ \\ U_{m 1}^{2}, \ldots, U_{m n}^{2}\end{array}\right]$

11. The system of equations is created which connect the complex amplitudes of the currents in the radiators and the coefficients of the partial HB for two targets.

For target 1:

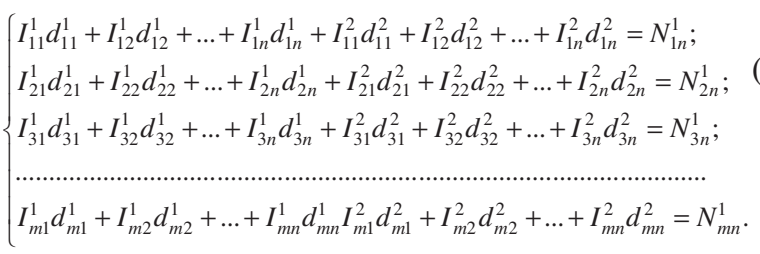

For target 2:

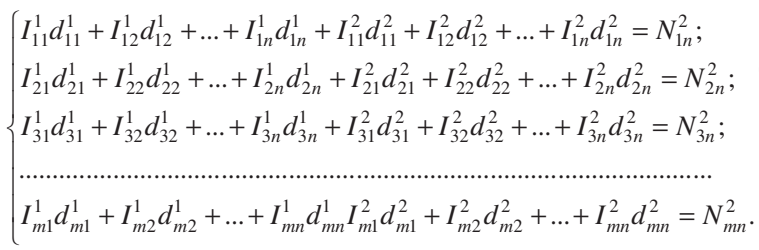


In these systems $d^{1,2}{ }_{m n}$ is the value $f\left(\theta_{m n}, \varphi_{n m}\right)$ for each target 1 and 2 from given directions $\theta_{m n}, \varphi_{n m}$, and $N_{m n}^{1,2}$ are the coefficients of partial diagrams of radiators $f(\theta \mathrm{mn}$, $\varphi n m)$ in angular sectors of observation maximum of $\mathrm{HB}$ EFDAA-DHFA for two targets 1 and 2 from the given directions $\theta_{m n}, \varphi_{n m}$.

\section{Conclusions}

All computational operations in the receiving channels of EFDAA-DHFA are common solutions finding of equation systems using the developed algorithm. It includes steps for defining partial phase centres of EFDAA-DHFA that are found by crossing out each of the members of the equations system to $m$ rows and $n$ column (row-column calculation algorithm).

In each of such a partial phase center of EFDAA-DHFA there are the ratio between the amplitudes of the currents $I_{m n}$ $\left(\theta_{m n}, \varphi_{n m}\right)$ and the phase offsetting between them $\psi_{X m}, \psi_{Y m}$ on $\mathrm{X}$ and $\mathrm{Y}$ axes of neighbouring radiators, with the help of determinants of matrices of each partial phase center of EFDAA-DHFA and matrices of determinants $I_{m n}\left(\theta_{m n}, \varphi_{n m}\right)$ and $\psi_{X m}, \psi_{Y m}$ on $X$ and $Y$ axes for each target 1 and 2 with the given directions $\theta_{m n}, \varphi_{m n}$.

The algorithm determines $\max U_{m n}\left(\theta_{m n}, \varphi_{m n}\right)$ for each target separately according to the formula:

$\max U_{m n}\left(\theta_{m n}, \varphi_{m n}\right)=\sum_{m=1}^{M} \sum_{n=1}^{N} U_{m n}^{1,2} \exp j\left(\psi_{X m n}^{1,2}+\psi_{Y m n}^{1,2}\right)$,

Amplitude $\max U_{m n}^{1}\left(\theta_{m n}, \varphi_{n m}\right)$ and $\max U_{m n}^{2}\left(\theta_{m n}, \varphi_{n m}\right)$, and phase angle coordinates are determined by the expressions:

$$
\begin{gathered}
\varphi_{m n}^{1}=\operatorname{arctg}\left(\frac{\psi_{y 1} d x_{1}}{\psi_{x 1} d y_{1}}\right), \theta_{m n}^{1}=\operatorname{arctg} \sqrt{\left(\frac{\psi_{x 1}^{2}}{\beta^{2} d x_{1}^{2}}\right)+\left(\frac{\psi_{y 1}^{2}}{\beta^{2} d y_{1}^{2}}\right)}, \\
\varphi_{m n}^{2}=\operatorname{arctg}\left(\frac{\psi_{y 2} d x_{2}}{\psi_{x 2} d y_{2}}\right), \theta_{m n}^{2}=\operatorname{arctg} \sqrt{\left(\frac{\psi_{x 2}^{2}}{\beta^{2} d x_{2}^{2}}\right)+\left(\frac{\psi_{y 2}^{2}}{\beta^{2} d y_{2}^{2}}\right)} .
\end{gathered}
$$

The formula for determining the variance of the quantization noise in the $\mathrm{ADC}$ of the microprocessor in accordance with $[13,14]$ has the form:

$$
\sigma_{\mathrm{q} . \text { error }}^{2}\left(\frac{I_{m n}^{1,2}}{I_{00}^{1,2}}\right)=\frac{1}{3}\left(\frac{D\left\{U_{m n}^{1,2}\right\}}{2^{B+1}}\right)
$$

where $U^{1,2}{ }_{m n}$ is the dynamic spectral band of input voltage of $\mathrm{ADC} ; B$ - is a digits of ADC.

The implementation of the developed algorithm allows to measure the angular coordinates when using EFDAA-DHFA in the circuit of MRS with the minimized dispersions (uncertainties) of measurements $\delta_{t}{ }_{t}, \delta_{t}{ }_{t}$ for each air object.

\section{References}

[1] I.R. Parhomey, J.M. Boiko, Features of objects radar systems ranging from low reflection surface. Herald of Khmelnytskyi National University, scientific journal, Technical Sciences 5/1 (2015) 194-201.

[2] I.R. Parhomey, J.M. Boiko, A.I. Eromenko, Development models of signal processing radar remote sensing. Herald of Khmelnytskyi National University, Scientific Journal, Technical Sciences 233/1 (2016) 7-23.

[3] L.D. Bakhrah, Y.G. Zelkin, Spravochnik po antennoy technike, Directory of antenna technology Ya.N. Field, Ye.G. Zelkin, Radiotechnique 1 (1997) (in Russian).

[4] J. Boiko, I. Parhomey, Automated control system radar work, Measurement and control in complex systems, $13^{\text {th }}$ IEEE International Conference, MCCS 2016, Vinnytsia, 2016, 109-111.

[5] R.C. Hansen, The segmented aperture synthetic aperture radar, IEEE Transaction Aerospace and Electronc System, SASAR V. AES-10/6 (2004) 800-804.

[6] S.V. Bearse, Computer-scanned radar eyes ice, Microwaves 17/12 (2001) 9-10.

[7] A. Iain, Multibeam synthetic aperture radar for global oceanography, IEEE Transactions on Antennas and Propagation 27/4 (2009) 535-538.

[8] C.A. Wiley, Syntetic Aperture Radar, IEEE Transaction on Aerospace and Electronic Systems, V. AES-21/3 (2005) 440-443.

[9] R.K. Raney, Synthetic aperture imaging radar and moving targets, IEEE Transactions on Aerospace and Electronic Systems, V. AES-7/3 (20010 499-505.

[10] C.W. Shervin, Some Early Developments in Synthetic Aperture Radar Systems, IRE Transaction, on Military Electronics V. MIL-6/2 (2006) 111-115.

[11] A.K. Lohrer, Improved azimuthal resolution of forward looking SAR by sophisticated antenna illumination function design, IEE Proceedings - Radar, Sonar and Navigation, V. 145/2 (2008) 128-134.

[12] V.A. Variuhin, Osnovy teorii mnogokanalnogo analysa, Basis of theory of multichannel analysis, Kyiv: WA PWO SW, 1993, (in Russian). 
[13] I.A. Kovalenko, B.F. Bondarenko, Osnovy postroeniya RLS, Basis of MRS forming, Kyiv: KWIRTU, 1976 (in Russian).

[14] V.I. Rudakov, Tsyfrovoy amplifazometr SVCH dla radiotechnicheskych system spetsialnogo naznachenyia, Digital amplifier of $\mathrm{HB}$ for radiotechnique system of special purpose, Izvestyia vyschych uchebnych zavedeniy - News of high educational institutions, Ser Radioelectronics, Military Radioelectronical Technology, Kyiv: NTUU, Kyiv Politechnical Institute 49/4 (2006) 6880, (in Russian).

[15] V.I. Rudakov, Troposfernye sistemu svyazy s adaptivnymi antennami, Troposcherical system of relation with the adaptive antennas, Kyiv: TSNII VVT VS of Ukraine, Avionika, 1999, (in Russian).

[16] O.G. Vendik, Antenny s nemekhanicheskim dvizheniem lucha: Vvedenie v teoriu, Antennas with the non-mechanical ray movement: Introduction of basis, Moscow: Sov. Radio, 1965, (in Russian).

[17] L.D. Bakhrah, Sintez izluchayucshych system, Synthesis of radiating system, Moscow: Sov. Radio, 1974, (in Russian).

[18] R.A. Monzingo, Adaptivnye antennye reshetky, Adaptive antenna arrays, V.A. Leksachenko, Moscow: Radio and Connection, 1986, (in Russian).

[19] S.Z. Kuzmin, Tsyfrovaya radiolokatsiya: Vvedenie v teoriu, Digital radiolocation: Introduction of basis, Kyiv, 2000, (in Russian). 